\title{
MODELLING AND NUMERICAL ANALYSIS OF ASSEMBLY SYSTEM
}

\author{
Zbigniew BUDNIAK* \\ *Faculty of Mechanical Engineering, Koszalin University of Technology, ul. Raclawicka 15-17, 75-612 Koszalin, Poland \\ zbigniew.budniak@tu.koszalin.pl
}

received 20 October 2014, revised 7 October 2015, accepted 9 October 2015

\begin{abstract}
The present articles covers a concept of the creation and testing of assembly systems with the use of modern CAD and CAE systems on the example of an assembly system designed for joining parts with circular surfaces that are fitted with positive clearance. The numerical investigations were based on the constructed spatial skeleton pattern of the system. The purpose of the simulation tests was to determine the impact of the measurement and angular inaccuracies of all the elements of the assembly system as well as the inaccuracy of the positioning of the robot's drives on the positioning accuracy of the parts joined taking into consideration the conditions of assembly in automatic assembly.
\end{abstract}

Key words: Modeling, Analysis, Assembly Manipulator, Chain Dimensional, Assembly System, CAD/CAE

\section{INTRODUCTION}

Issues related to the specificity of modeling and kinematic analyses of technical systems were presented in papers Bil, (2011, 2012, 2013); Chen et al. (2014); Liuet et al. (2012), Kacalak et al. (2015). The present article covers a method to create and test assembly systems that comprise an assembly robot, a holder to fasten the body with a fitted opening, a gripper and the parts to be combined.

The essential part of the work presented included a verification of the correctness of the spatial model developed of the assembly system, development of the parametric models of the parts and the assembly, conducting a kinematic analysis (Budniak, 2012) and its visualization including its animation. SolidWorks software was used for the purpose of parametric solid modelling of the mounting seat. Kinematic calculations and analyses were conducted with the use of SolidWorks Motion software (Chang, 2011).

Special attention needs to be drawn to the possibility of conducting an analysis of the accuracy of the mutual position of the assembly system elements based on spatial dimension chains that are of a key importance in their designing. The task of the identification of dimension chains is strenuous and there is a high risk of making an error, especially in the case of mechanisms with a large number of parts with complex geometries. Owing to the use of spatial dimension chains, which are recorded as models in $\mathrm{CAD} / \mathrm{CAE}$, it is possible to conduct a verification of the conceptual spatial design of the assembly system, an analysis of the mutual positions of the elements combined in the technological process of assembly (Żurek et al., 2013; Tabara et al., 2013), an analysis of the dimensional and shape accuracy of the constituent elements of the assembly system as well as an analysis of clearances in movable connections (Storch et al., 2007; Zebrowski et al., 2009; Ahn et al., 2013).

\section{CHAPTER TITLE}

The process of automatic assembly could not proceed correctly without the required accuracy of the position of the parts combined at each stage (Yun et al., 2010; Zhu et al., 2013; Tabara et al., 2013). This follows particularly from the seizure conditions of the elements combined, which in the majority of cases are the conditions of automatic assembly (Budniak, 2014; Huang, 2005). The mutual position of the parts combined is particularly important in the moment of their positioning (Fig.1a) and centering (Fig.1b).

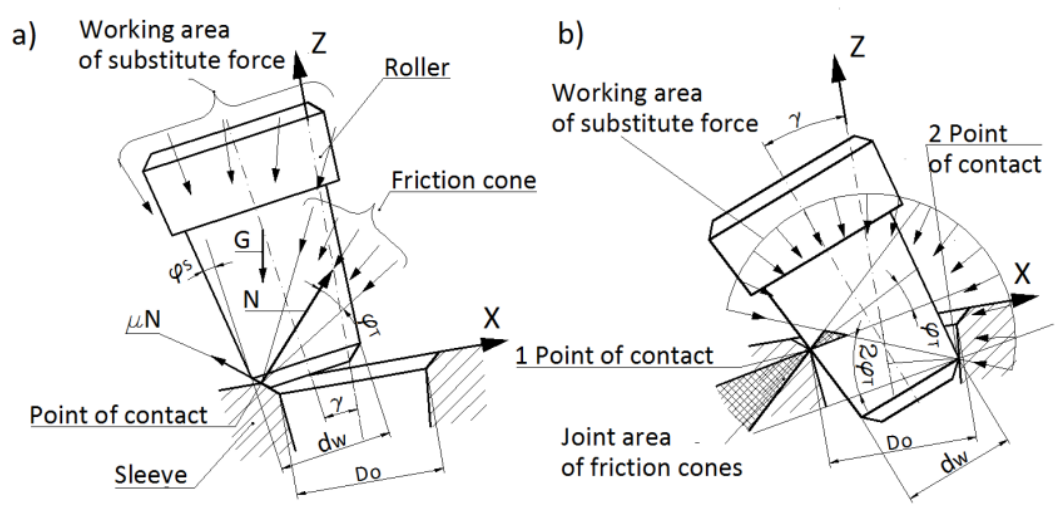

Fig. 1. The position of combined parts, where: a) positioning, b) centering 
The first stage of the combination of the parts is characterized by a single-point contact of the elements combined. Their seizure occurs only when the direction of the substitute assembly force passes through the contact point of the parts and it is simultaneously inside the cone of friction $2 \varphi T$; cf. Fig.1a. It is evident from the figure that the probability of the parts combined being seized is minute; however, it grows when angle $\varphi T$ is increased. The probability of the parts combined being seized during their displacement: both on the surfaces of phases and on the frontal surface of the sleeve is small.

The second stage of combining parts is substantially different from the first one, as the surfaces that are matched contact one another in two points (Fig.1b). In order to determine the seizure conditions of the parts to be combined, a mechanical engineering law was accepted, according to which: if multiple forces and moments act on an element, they can be reduced to one substitute force, while it can be found that the roller that is inserted is in standstill when the substitute assembly force cuts the common area of friction cones.

Seizure occurs when the substitute force that acts on the roller that is inserted cuts the equilibrium area that is the common area of friction cones (Fig. $1 \mathrm{~b}$ ). This area is limited by a polygon, whose vertex coordinates are intersection points of the forming outline friction cones $2 \varphi T$, where the value of this angle is calculated from the following formula:

$\varphi_{T}=\operatorname{arctg} \mu$

where: $\mu$ - friction coefficient value

At the moment of centering, the parts to be combined ought to take such a position so that with any of their dimensions that are within tolerance limits there should not occur any seizure of these. Hence, the conditions of automatic assembly are as follows:

$\gamma \leq \gamma_{\text {perm }}, \omega_{\Delta} \leq \omega_{\Delta}$,

$$
\begin{aligned}
& \gamma_{\text {perm }}=\arccos \left(\frac{d_{w}}{D_{o}} * \cos \varphi_{T}\right)-\varphi_{T}, \\
& \omega_{\Delta}=\left(D_{o}-d_{w} \cdot \cos \gamma_{\text {perm }}\right) / 2 .
\end{aligned}
$$

Owing to the dependences present, it is possible to reveal ways to obtained the required positional accuracy. If Condition (2) is fulfilled, everything depends from what the direction will be of the substitute assembly force. This direction will chiefly depend from the accepted assembly diagram, the constructional details of the assembly mechanisms, the rigidity of the assembly system, the misalignment of the axes of the surfaces matched etc.

\section{SPATIAL MEASUREMENT CHAIN OF THE ASSEMBLY SYSTEM}

In the present study, a numerical analysis was conducted of the assembly system for combining parts of the roller-sleeve type as presented in Fig. 2. This system constitutes the basis of a robotic mounting seat, which consists of an assembly robot and peripheral instrumentation. The $R R R P R R$ assembly robot possesses six degrees of freedom: five rotational couples and one sliding couple.

In order to guarantee the mutual position of the axes of the elements to be combined, points $Q_{s}$ and $P_{h}$ with a specific accuracy, one needs to determine above all the mutual position of the elements of the assembly system. Value $r_{\Delta}$, which determines the error of the mutual position of nodal points $Q_{s}$ and $P_{h}$, depends from the accuracy of the relative position of the arms of the assembly robot $\left(r_{1}, r_{2}\right.$ and $\left.r_{3}\right)$, servo-motor $\mathrm{s}$, gripper e with the gripping part $\mathrm{c}$, the base of the robot $p$, the elements to be combined and the accuracy of their workmanship. The determination of the closing link $r_{\Delta}$ was based on the solution of the spatial measurement link (Fig. 2).

where for cylindrical parts to be combined (conical surface angle $\left.\varphi_{S}=0^{0}\right)$ :

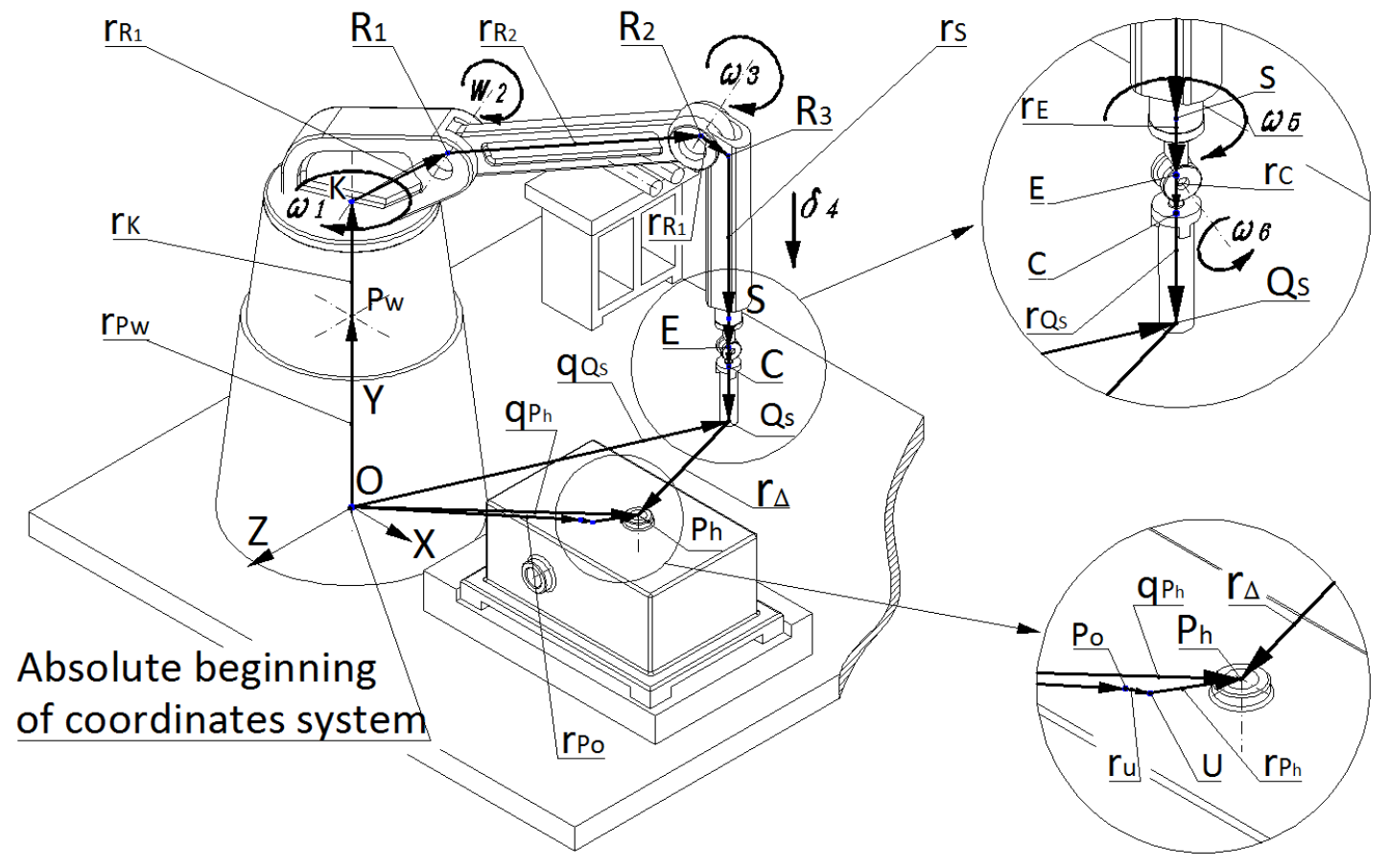

Fig. 2. Location of the assembly system components 
In the general case, the value of the closing link $r_{\Delta}$, the spatial measurement chain of the assembly system, is calculated from Formula (4):

$r_{\Delta}=q_{Q_{S}}-q_{P_{h}}=r_{P_{w}}+r_{K+} r_{R_{1}}+r_{R_{2}}+r_{R_{3}}+r_{S}+r_{E}+$

$r_{C}+r_{P_{o}}+r_{U}+r_{P_{h}}$,

where: $q_{Q_{S}}, q_{P_{h}}$ - vectors that determine the position of points $Q_{s}$ and $P_{h}$ in relation to the global system of coordinates $O X Y Z$, $r_{P_{w}}, r_{P_{o}}$ - vectors that determine the position of nodal points $P_{w}$ and $P_{O}$ in relation to the global system of coordinates $O X Y Z$, $r_{K}, r_{R_{1}}, r_{R_{2}}, r_{R_{3}}, r_{S}, r_{E}, r_{C}, r_{Q_{S}}, r_{U}, r_{P_{h}}$ - vectors that determine the position of nodal points $K, R_{1}, R_{2}, R_{3}, S, E, C, Q_{S}, U, P_{h}$ that belong to the individual constituent elements of the assembly system in relation to their local systems of coordinates.

\section{MODELLING OF SPATIAL MEASUREMENT CHAIN}

A description of the assembly system can be considered as a description of the relative position of the local systems of coordinates related to the individual links of the measurement chain: to the reference link (base), the so-called global system $O X Y Z$ is attributed (Fig. 2). Such an approach to the description of this system orders and formalizes its modeling both in the area of the kinematics and dynamics of the assembly process.

At every moment, the movable elements of the assembly system accept a specific position in relation to the base and in relation to one another. When making an analysis of the position of this system, the determination is of particular importance of the mutual position of points $Q_{s}$ and $P_{h}$ for the specified position of the remaining elements of the system. To the individual links of the assembly system, the following were attributed: the absolute system of coordinates $O X Y Z$ related to the immovable base as well as local systems of coordinates connected with main and auxiliary assembly bases of the remaining components.

In the part of the assembly system (base $p$, the body of the assembly robot $\mathrm{k}$, the arms of the assembly robot $r_{1}, r_{2}, r_{3}$, servo-motor s, gripper e and its gripping part c and roller w), point $Q_{s}$ was selected, whose position is in the local system of coordinates $O_{B_{Q_{S}}} X_{B_{Q_{S}}} Y_{B_{Q_{S}}} Z_{B_{Q_{S}}}$ was determined as vector $Q_{S_{E}}$. In a particular case, the position of the centre of the roller with the length being equal to $l_{o}$, on its frontal surface, the following is calculated:

$Q_{S_{E}}=\left[\begin{array}{c}0 \\ -l_{o} \\ 0\end{array}\right]$.

The same point is described with vector $Q_{s}$, which determines its position in the absolute system $O X Y Z$ :

$Q_{S}=R_{P_{w}} \cdot Q_{S_{P_{w}}}+T_{P_{w}}$,

where:

$Q_{S_{P_{w}}}=R_{K} \cdot Q_{S_{K}}+T_{K}$,

$Q_{S_{K}}=R_{R_{1}} \cdot+T_{R_{1}}$,

$Q_{S_{R_{1}}}=R_{R_{2}} \cdot Q_{S_{R_{2}}}+T_{R_{2}}$,

$Q_{S_{R_{2}}}=R_{R_{3}} \cdot Q_{S_{R_{3}}}+T_{R_{3}}$,

$Q_{S_{R_{3}}}=R_{S} \cdot Q_{S_{S}}+T_{S}$,
$Q_{S_{S}}=R_{E} \cdot Q_{S_{E}}+T_{E}$,

$Q_{S_{C}}=R_{C} \cdot Q_{S_{C}}+T_{C}$,

$R_{P_{w}}, R_{K}, R_{R_{1}}, R_{R_{2}}, R_{R_{3}}, R_{S}, R_{E}, R_{C}$ - matrices of rotation that determine the rotation of the local systems of coordinates, whose beginnings are in points $P_{w}, K, R_{R_{1}}, R_{R_{2}}, R_{R_{3}}, S, E$ and $C$ around their axes; $T_{P_{w}}, T_{K}, T_{R_{1}}, T_{R_{2}}, T_{R_{3}}, T_{S}, T_{E}, T_{C}$ - vectors that describe the displacements of the local systems of coordinates.

In a part of the assembly system (base $p$, gripper $u$ and the body with opening 0 into which roller w is inserted), point $P_{h}$ was selected, whose position in the local system of coordinates $O_{B_{U}} X_{B_{U}} Y_{B_{U}} Z_{B_{U}}$ was determined as a vector:

$P_{h_{U}}=\left[\begin{array}{c}X_{B_{U}} \\ Y_{B_{U}} \\ Z_{B_{U}}\end{array}\right]$.

The same point is described with vector $P_{h}$ that determines its position in the absolute system $O X Y Z$ :

$P_{h}=R_{P_{o}} \cdot P_{h_{P_{o}}}+T_{P_{o}}$

where:

$P_{h_{P_{o}}}=R_{U} \cdot P_{h_{U}}+T_{U}$,

$R_{P_{o}}, R_{U}$ - the matrix of rotation that determines the rotation of the local systems of coordinates, whose beginnings are located in points $P_{o}$ and $R_{U}$ around their axes.

\section{MODELLING OF A VIRTUAL MODEL OF THE ASSEMBLY SYSTEM}

The first stage of the construction of the virtual model of the mounting seat including the assembly system was the creation of models that contain the constructive geometry of its individual parts. This geometry is formed by planes, axes and constructional points as well as the beginning of the local systems of coordinates. The parameters of constructive geometry, which determines the position of the local systems of coordinates, their axes and points were written in the form of modeling variables. The values of these variables correspond to the elements of the matrix of rotation of the vectors described in Equations (5-16).

While being based on the model of the base of the assembly system, the relations were defined that occur between the remaining elements of the assembly. For the purpose of a unique determination of the position of the individual elements of the mechanism, the assembly bases that are adjacent to one another of the components to be combined were used. The view of the virtual mounting seat including the assembly system is shown in Fig. 2.

\section{KINEMATIC SYSTEM OF THE ASSEMBLY SYSTEM}

The structure of the assembly system shown in Fig. 2 presents the movement capacity of the RRRPRR assembly robot. The movements for the individual axes are obtained with the aid of drives that transfer kinematic energy to the mobile members.

In order to determine the position and orientation of gripper $e$ with roller $w$ inserted in relation to the axis of the opening in the body, into which it is inserted, one needs to assign rectangular systems of coordinates to the links of the assembly system 
in accordance with the previously presented Formulae (5-16). From the point of view of the assembly process, the determination is the most important of the mutual position of the axis of the elements to be combined: of the roller $w$ and the axis of the opening in the body $o$. Hence, in further studies, the position and orientation of the roller will be analyzed. The roller $w$ is permanently connected with the auxiliary assembly bases of the gripping part $c$ of the gripper $e$, and its symmetry axis includes point $Q_{S}$, whose vector of position is described with Formula (6).

In the simulation model of the SolidWorks Motion software, in order to determine the dependencies between the configuration coordinates and the base coordinates $\left(\Omega_{1}, \Omega_{2}, \Omega_{3}, \delta_{4}\right)$ of the position and orientation of the roller in $\left(\Omega_{5}, \Omega_{6}\right)$, temporary $3 D$ connections were introduced that determine the position of the assembly system and its elements in the mounting seat in the starting and final positions.

One needs to take note of the fact that the local system of coordinates $O_{B_{E}} X_{B_{E}} Y_{B_{E}} Z_{B_{E}}$ with the roller w inserted is connected with the working space and it constitutes the basic system when determining the mutual positions of the elements to be combined. The system $O_{B_{R_{1}}} X_{B_{R_{1}}} Y_{B_{R_{1}}} Z_{B_{R_{1}}}$ is connected with the link $r_{1}$ and it can rotate solely by the angle $\Omega_{1}$ in relation to the axis $X_{B_{R_{1}}}$. The local systems of coordinates $O_{B_{R_{2}}} X_{B_{R_{2}}} Y_{B_{R_{2}}} Z_{B_{R_{2}}}$ and $O_{B_{R_{3}}} X_{B_{R_{3}}} Y_{B_{R_{3}}} Z_{B_{R_{3}}}$ are connected with the $r_{1}$ and $r_{2}$ links. For these systems, rotations are possible by the angle $\Omega_{2}$ in relation to the axis $Z_{B_{R_{2}}}$ and around the axis $Z_{B_{R_{3}}}$. The system of coordinates $O_{B_{S}} X_{B_{S}} Y_{B_{S}} Z_{B_{S}}$ of the linear servo-motor $s$ is displaced in the direction of the gripper $e$ by value $\delta_{4}$. The system of coordinates $O_{B_{E}} X_{B_{E}} Y_{B_{E}} Z_{B_{E}}$ of the gripper $e$ is placed in the central part of the gripper and is determined by rotations by the angle $\Omega_{5}$ in relation to the axis $Z_{B_{E}}$ and $\Omega_{6}$ around the axis $Y_{B_{E}}$.

Forcing of the relative motion of the roller in order to perform a simulation of the motion was obtained through the use of virtual engines that perform a rotary motion with the following velocities: $\omega_{1}, \omega_{2}, \omega_{3}, \omega_{4}$ and $\omega_{6}$. In the position of the positioning of the roller, the linear engine $v_{1}$ was started that forces the translational motion of the gripper $e$.

\section{POSITION AND ORIENTATION OF THE ASSEMBLY ROBOT}

In order to conduct simulation tests, the assembly system was selected that is presented in Fig. 2. To describe the movement path of the roller that is inserted in the opening in the sleeve, $P_{h}$ and $Q_{s}$ points were selected. It was accepted that these points are located on the symmetry axes of the roller and the sleeve on their frontal surfaces. The positions of these points are sought on their trajectories that are the result of the constraints imposed by the individual member and kinematic pairs of the assembly system.

Forcing of the relative motion of the roller inserted, in order to perform a simulation of the movement, was obtained by the use of the virtual drives of the individual elements of the assembly robot in accordance with the motion parameters contained in Tab.1.

The numerical analysis of the relative position of the elements of the assembly system was conducted for the centre of the roller $Q_{s}$ inserted in relation to the motionless centre of the sleeve $P_{h}$. The distance between these centers $\omega_{\Delta}$ was calculated from the following formula:

$\omega_{\Delta}=\sqrt{\Delta_{X}^{2}+\Delta_{Z_{U}}^{2}}$,

where:

$\Delta_{X}=X_{Q_{s}}-X_{P_{h}}, \Delta_{Z}=Z_{Q_{s}}-Z_{P_{h}}$.

Tab. 1. Parameters motion of the robot assembly

\begin{tabular}{|c|c|c|c|c|c|c|}
\hline \multirow[b]{2}{*}{$\begin{array}{l}\text { Virtual } \\
\text { engine }\end{array}$} & \multicolumn{2}{|c|}{ Speed } & \multicolumn{2}{|c|}{ Displacement } & \multicolumn{2}{|c|}{ Time } \\
\hline & $\begin{array}{c}\begin{array}{c}\text { rotatio } \\
\text { nal }\end{array} \\
{\left[{ }^{\circ} / s\right]}\end{array}$ & $\begin{array}{l}\text { linear } \\
{[\mathrm{m} / \mathrm{s}]}\end{array}$ & $\begin{array}{c}\text { angular } \\
{\left[^{\circ}\right]}\end{array}$ & $\begin{array}{l}\text { linear } \\
{[\mathrm{mm}]}\end{array}$ & $\begin{array}{c}\text { comme } \\
\text { nncem } \\
\text { ent of } \\
\text { motion } \\
\text { [s] }\end{array}$ & $\begin{array}{c}\text { duration } \\
\text { of } \\
\text { motion } \\
\text { [s] }\end{array}$ \\
\hline$\omega_{1}$ & 150 & - & 90 & - & 0.2 & 0.6 \\
\hline$\omega_{2}$ & 150 & - & 30 & - & 0 & 0.2 \\
\hline$\omega_{3}$ & 150 & - & 30 & - & 0.2 & 0.2 \\
\hline$v_{4}$ & - & 0.6 & - & 120 & 0.8 & 0.2 \\
\hline$\omega_{5}$ & 150 & - & 45 & - & 0.2 & 0.3 \\
\hline$\omega_{6}$ & 180 & - & 90 & - & 0.1 & 0.5 \\
\hline
\end{tabular}
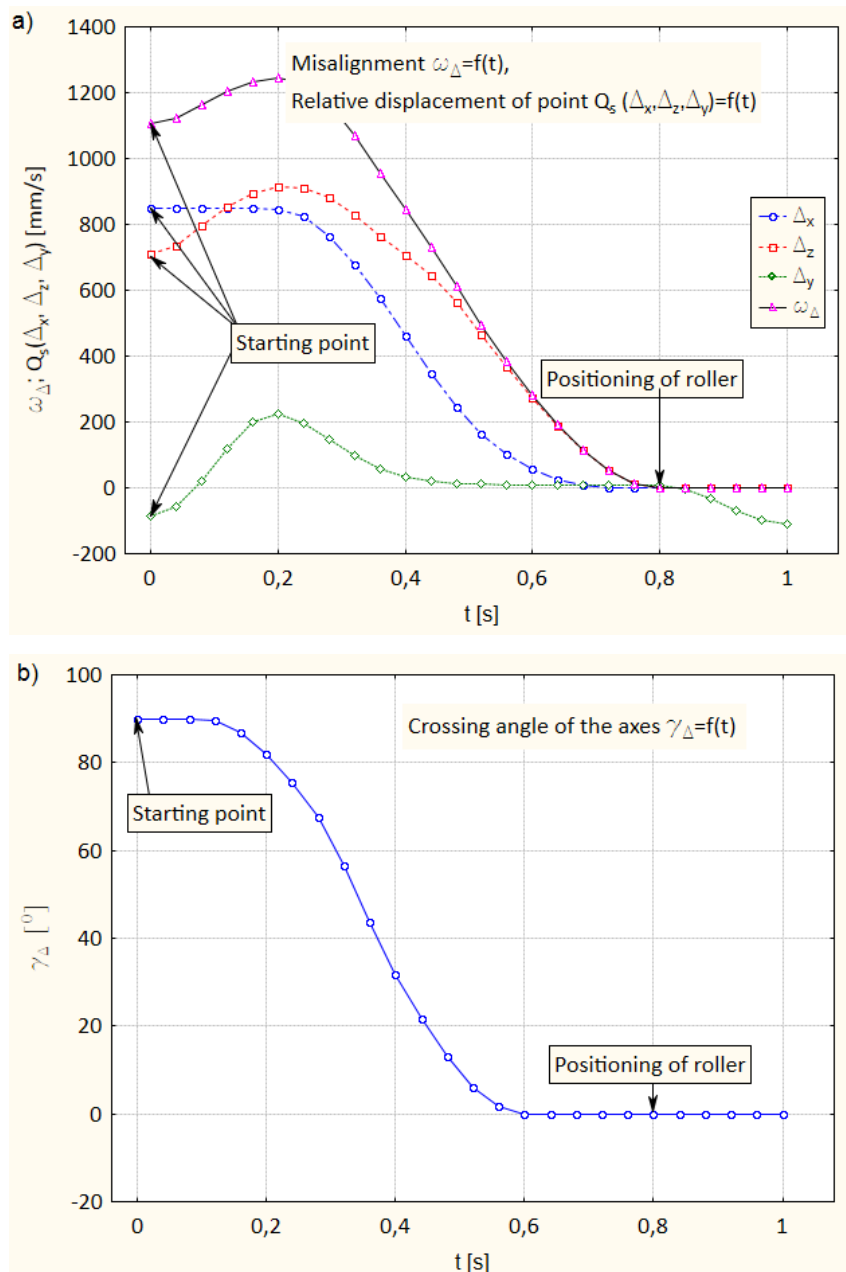

Fig. 3. Misalignment and crossing angle of the axes of the parts to be combined

The simulation model developed facilitated an easy determination of the trajectory for the known position of the point $Q_{s}$ 
in the global system of coordinates $O X Y Z$. Fig. 3 presents the values of misalignment in the global system of coordinates $O X Y Z$ and the warping angles of their axes $\gamma_{\Delta}$ of the parts to be combined along the assembly path, where $\Delta y$ specifies the change of the distance between the frontal surfaces of the roller and of the sleeve. Numerical tests were conducted for the assembly system, where the individual elements are performed with absolute accuracy. This means that the value of the closing link at the moment of positioning is $\omega_{\Delta}=0 \mathrm{~mm}$ (Fig. 3a) and the crossing angle of the axes $\gamma_{\Delta}=0^{\circ}$ (Fig. 3b).

Fig. 4 presents the formation of the misalignment $\omega_{\Delta}$ and the warping angles $y_{\Delta}$ of the axes of the parts to be combined depending from the linear $E L$ and angular $E A$ accuracy of the elements of the assembly system and the positioning accuracy $E P$ of the virtual engines. In the simulation tests, assembly was accepted by means of the complete variability method with identical workmanship tolerance for all the links of the measurement chain. For linear measurements, a workmanship accuracy was accepted of $8 \mu \mathrm{m}$, while for angular measurements, this accuracy amounted to $0.02^{\circ}$. The positioning accuracy of the virtual rotating engines was $\omega_{\Delta_{i}}= \pm 0.02^{\circ}$, and for the virtual translational servomotor, this was $\partial_{\Delta_{4}}= \pm 0.02 \mathrm{~mm}$.
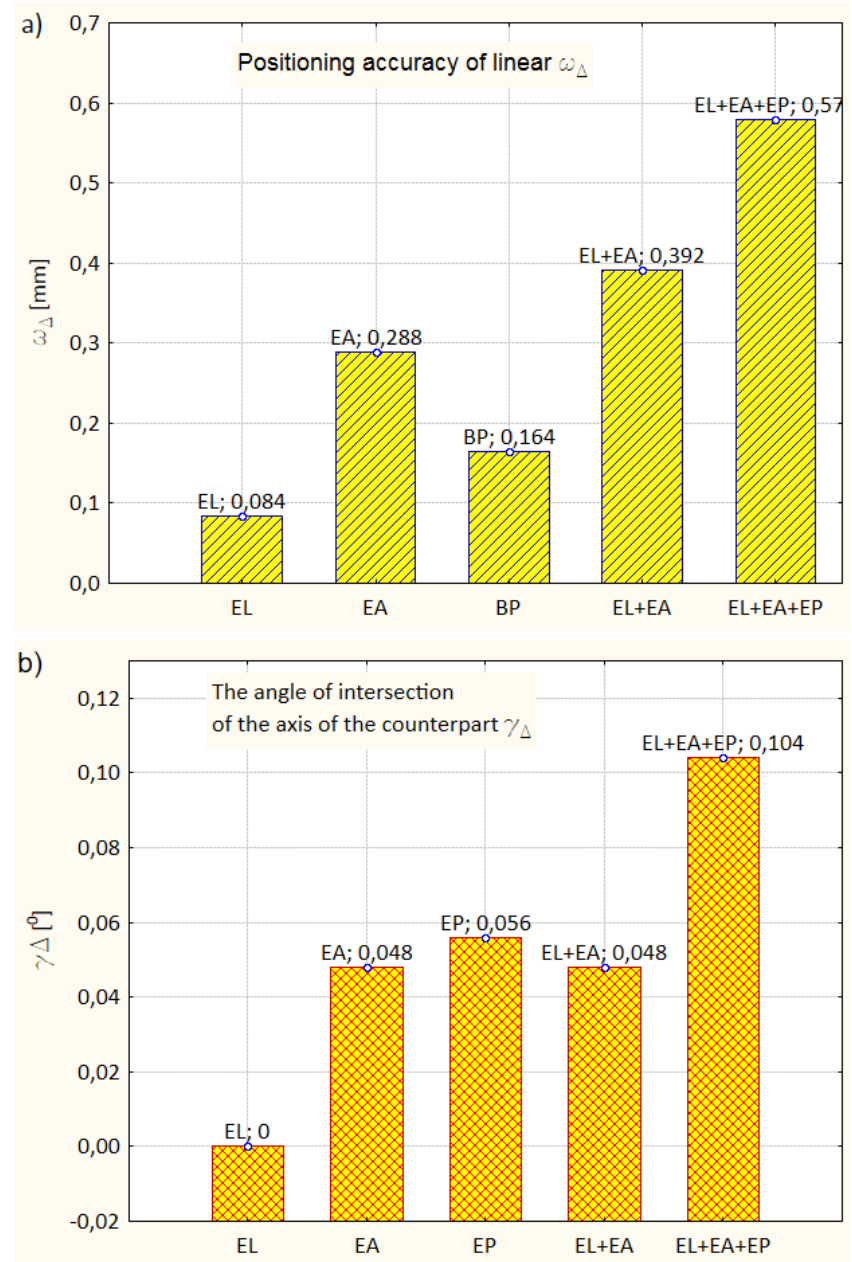

Fig. 4. Misalignment and crossing angle of the axes of the parts to be combined

The results presented of the simulation tests for the summary constituent errors $(E L+E A+E P)$ show that the greatest misalignment is $\omega_{\Delta}=0.579 \mathrm{~mm}$, and the warping angle is $y_{\Delta}=0.104^{\circ}$.
Owing to the values found of positioning errors, it is possible to verify the possibility of an automatic assembly of parts of the roller-sleeve type depending from the mountability conditions that are described with Equations (2 and 3). For the system under analysis, with the friction coefficient between the surfaces of the parts to be combined being $\mu=0.15$, automatic assembly will be possible solely for clearances in combination:

\section{CONCLUSIONS}

This article presents the potential of the present-day CAD/CAE systems for modeling and a kinematic analysis of a virtual assembly system. The simulation model developed makes it possible to conduct numerical analyses of the assembly system based on a spatial measurement chain. Among others, this allows one to:

- investigate the accuracy of positioning and mountability conditions in automatic assembly taking into account the workmanship accuracy of the individual parts and units of the assembly system;

- determine the impact of the accuracy of the motion of those engines that drive the movable elements of the assembly system on the positioning accuracy, which will facilitate an optimal selection of the assembly robot (Żurek et al., 2011; Kuzmierowski, 2010).

The virtual simulation model constructed of an assembly system with the use of CAD/CAE computer technology takes into account numerous parameters of real assembly. The advantages that follow from the analytical model presented are huge as they allow a thorough assessment of the designs made of technical systems. The results presented in the study of simulation tests contain an illustration only of selected factors that have an influence on the work of the assembly system.

\section{REFERENCES}

1. Ahn K., Cho D. (2013), Proposition for a Volumetric Error Model Considering Backlash in Machine Tools, The International Journal of Advanced Manufacturing Technology, Vol. 15, Issue 8, 554-561.

2. Bil T. (2010), Kinematic analysis of a universal spatial mechanism containing a higher pair based on tori. Mechanism and Machine Theory. Vol.46, Issue 4, 412-424.

3. Bil T. (2012), Analysis of the Bennett linkage in the geometry of tori. Mech. \& Mach. Theory, Elsevier, Vol.53, 122-127.

4. Budniak Z. (2013), Modeling of spatial dimension chains of mounting systems using techniques CAD/CAE. Pomiary Automatyka Kontrola, vol. 59, 652-655.

5. Budniak Z. (2014), Mountability parts of machine with rotating surface, fitted with positive clearance. Zeszyty Naukowe Politechniki Śląskiej. Transport, 83, 29-38.

6. Budniak Z., Bil T. (2012), Simulation of the movement of four-bar spatial linkage. International Journal of Applied Mechanics and Enginering, vol.17, No.4, 723-732.

7. Chang K-H. Motion Simulation and Mechanism Design with SolidWorks Motion 2011. Schroff Development Corporation, 1-19.

8. Chen Y., Xie F., Liu X., Zhou Y. (2014), Error modeling and sensitivity analysis of a parallel robot with SCARA (selective compliance assembly robot arm) motions. Chinese Journal of Mechanical Engineering, Vol. 27, Issue 4, 693-702.

9. Huang S., Tsai J. (2005), Robotic automatic assembly system for random operating condition, The International Journal of Advanced Manufacturing Technology, Vol. 27, Issue 3, 334-344. 
10. Kacalak W., Majewski M., Budniak Z. (2015), Worm Gear Drives With Adjustable Backlash, Journal of Mechanisms and Robotics, Vol.8, Issue 1, p.7.

11. Kuźmierowski T. (2010), Analysis of the geometrical accuracy of the positioning of the effector of the parallel manipulator spatial type triplanar. Acta Mechanica et Automatica, Vol.4 no.1, 56-60.

12. Liu J., Zhang Z., Liu Y. (2012), Universal mechanism modeling method in virtual assembly environment. Chinese Journal of Mechanical Engineering, Vol. 25, Issue 6, 1105-1114.

13. Liu Z., Nakamura T. (2007), Combination of robot control and assembly planning for a precision manipulator. The International Journal of Advanced Manufacturing Technology, Vol. 31, Issue 7-8, 797-804.

14. Storch B., Wierucka I. (2007), Optyczne pomiary zarysów powtarzalnych wykorzystaniem technik przetwarzania obrazu, Acta Mechanica et Automatica, Vol.1, nr 2, 59-62.

15. Tabara I., I. Dugaesescu I. (2013), The Analysis of Part Positioning and Orientation in Robotic Assembly by Insertion. New Trends in Mechanism and Machine Science Mechanisms and Machine Science, Vol. 7, 509-517.

16. Yun Y., Li Y. (2010), Design and analysis of a novel 6-DOF redundant actuated parallel robot with compliant hinges for high precision positioning. Nonlinear Dynamics, Vol. 61, Issue 4, 829-845.

17. Żebrowski-Kozioł M., Tarnowski W. (2009), Diagnozowanie stanu luzów w połączeniach przegubowych przedniego zawieszenia samochodu, Acta mechanica et automatica, vol.3 no.2, 122-124.

18. Zhu W., Mei B., Yan G., Ke Y. (2014), Measurement error analysis and accuracy enhancement of $2 \mathrm{D}$ vision system for robotic drilling. Robotics and Computer-Integrated Manufacturing, Vol. 30, Issue 2, 160-171.

19. Żurek J., Ciszak O., Cieślak R., Suszyński M. (2011), Assessment and choice of an industrial robot with the use of AHP method. Archives of Mechanical Technology and Automation, Vol. 31, nr 2, 201-211.

20. Żurek J., Wiśniewski M. (2013), The methodology and research conditions of Fanuc M-16iB industrial robot positioning accuracy and repeatability. Technologia i Automatyzacja Montażu, 2013, Vol.1, 31-34.

This project has been funded by the National Science Centre on the basis of the decision no. DEC-2012/05/B/ST8/02802. 